\title{
Structures of the Molecular Components in DNA and RNA with Bond Lengths Interpreted as Sums of Atomic Covalent Radii
}

\author{
Raji Heyrovska*
}

\author{
Institute of Biophysics of the Academy of Sciences of the Czech Republic, Královopolská 135, 61265 Brno, Czech \\ Republic
}

\begin{abstract}
The author has found recently that the lengths of chemical bonds are sums of the covalent and or ionic radii of the relevant atoms constituting the bonds, whether they are completely or partially covalent or ionic. This finding has been tested here for the skeletal bond lengths in the molecular constituents of nucleic acids, adenine, thymine, guanine, cytosine, uracil, ribose, deoxyribose and phosphoric acid. On collecting the existing data and comparing them graphically with the sums of the appropriate covalent radii of $\mathrm{C}, \mathrm{N}, \mathrm{O}, \mathrm{H}$ and $\mathrm{P}$, it is found that there is a linear dependence with effectively unit slope and zero intercept. This shows that the bond lengths in the above molecules can be interpreted as sums of the relevant atomic covalent radii. Based on this result, the author has presented here the atomic structures of the above molecules in terms of the atomic radii (for the first time).
\end{abstract}

\section{INTRODUCTION}

Following the exciting discovery [1] of the molecular structure of nucleic acids, the question of the skeletal bond lengths in the molecules constituting DNA and RNA has continued to be of interest. The author was enthused to undertake this work by the recent findings [2a, b] that the length of the chemical bond between any two atoms or ions is, in general, the sum of the radii of the atoms and or ions constituting the bond. The additivity of radii was also found to hold for the hydration bonds [3a] with ions in aqueous solutions as well as for the lengths of the hydrogen bonds [3b] in the Watson-Crick [1b] base pairs of nucleic acids and in many other inorganic and biological compounds.

Pauling [4] dealt with the bond lengths in the purines: adenine $(A)$ and guanine $(G)$ and the pyrimidines: thymine (T), cytosine (C) and uracil (U), but he did not interpret the bond lengths in these molecules in terms of the covalent radii of the atoms, although he considered covalent bond lengths as sums of the covalent radii of the atoms. This article shows that the existing bond lengths [4-7] (see Tables 1 and 2) in the above molecules and in ribose, deoxyribose and phosphoric acid, abbreviated here as $\mathrm{Ri}, \mathrm{De}$ and $\mathrm{Ph}$ respectively, correspond to the sums of the appropriate covalent radii of the five atoms, carbon, nitrogen, hydrogen, oxygen and phosphorus.

\section{ATOMIC COVALENT RADII AND THE CN BOND LENGTH IN ADENINE}

It was noticed by Pauling [4] that in all the bases, A, T, $\mathrm{C}, \mathrm{G}$ and $\mathrm{U}$, (see Fig. (1)), the CC bond length is around 1.40 $\AA$ and the $\mathrm{CN}$ bond length has values around $1.32 \AA$ and $1.36 \AA$. A survey of the existing bond length data [4-7] (see Tables 1 and 2), shows that the $\mathrm{CC}, \mathrm{CN} \mathrm{CH}, \mathrm{NH}, \mathrm{CO}, \mathrm{OH}$ and $\mathrm{PO}$ bonds in the above molecules do have some essentially fixed bond lengths, in addition to the three considered by Pauling.

*Address correspondence to this author at the Institute of Biophysics of the Academy of Sciences of the Czech Republic, Královopolská 135, 61265 Brno, Czech Republic; E-mail: rheyrovs@hotmail.com
For the interpretation here of these bond lengths, the atomic covalent radii, defined [4a] as $R_{\text {cov }}=d(A)=d(A A) / 2$, where $d(A A)$ is the covalent bond length between two atoms (A) of the same kind, have been used. In [4b], these radii are referred to as bonding atomic radii. Since $R_{\text {cov }}$ is half the inter-atomic distance, it is actually a distance although written with an R. Considering carbon, Pauling [4] points out two types of single bond radii for $\mathrm{C}$ : the aliphatic single bond covalent radius of $0.77 \AA$ (as in cubic diamond), and the aromatic single bond (involved in resonance) covalent radius of $0.72 \AA$ (as in hexagonal graphite with delocalized charge). The covalent double bond radius of $C$, defined [4] as half the $\mathrm{C}=\mathrm{C}$ double bond length, is $0.67 \AA$. Note that 0.72 $=(0.77+0.67) / 2$, as if it is a midway 1.5 bond! In general, the covalent double bond radii of atoms are less than those for single bonds [4]. The above three $\mathrm{C}$ (with the subscripts, s.b: single bond, res: aromatic resonance bond and d.b: double bond) are represented by circles of radii $R_{\text {cov }}$ in Fig. (2). Similarly, in Fig. (2), $\mathrm{R}_{\text {cov }}$ for $\mathrm{N}_{\text {s.b }} \& \mathrm{~N}_{\text {d.b }}$ and $\mathrm{O}_{\text {s.b }} \& \mathrm{O}_{\text {d.b }}$ are halves of the single bond and double bond lengths of $\mathrm{N}$ and $O$ respectively, $R_{\text {cov }}$ for $H$ is half the $H H$ single bond distance [4] and $R_{\text {cov }}$ for $P$ was obtained by subtracting $R_{\text {cov }}$ of $\mathrm{O}(\mathrm{I})$ from the $\mathrm{PO}(\mathrm{I})$ bond length [ 4-7].

An interesting observation from the bond length data in [4-7] assembled in Table 1 is e.g., that the $\mathrm{CN}$ bond lengths in the aromatic ring in adenine (see col. 2, Table 1) are the same $[1.34(+/-) 0.02 \AA]$ irrespective of whether they pertain to single (N1-C2 and N3-C4) or double (C2-N3 and C6-N1) bonds. This is similar to the finding in [4] that in the case of benzene, all the six CC bonds are of length $1.39(+/-) 0.01 \AA$ (with equal assigned [4] bond order of 1.5, due to resonance), although represented by three alternating single and double bonds. This $\mathrm{CC}$ bond length in the aromatic ring was interpreted $[2 \mathrm{a}]$ as the sum $(0.72+0.67 \AA)$ of the radii for $\mathrm{C}_{\text {res }}$ and $\mathrm{C}_{\mathrm{d} . \mathrm{b}}$ (see Fig. (2)). Similarly, in adenine, the $\mathrm{CN}$ bond distance of $1.34 \AA$ is the sum of $R_{\text {cov }}$ of $C_{\text {res }}(=0.72)$ and $\mathrm{N}_{\mathrm{d} . \mathrm{b}}(=0.62)$. 


\section{Thymine (T) Adenine (A) Cytosine (C) Guanine (G) Uracil (U)}<smiles>Cc1cn(Cl)c(=O)[nH]c1=O</smiles><smiles>Nc1ncnc2c1ncn2Cl</smiles><smiles>[CH]n1ccc(N)nc1=O</smiles><smiles>Nc1nc2c(ncn2Cl)c(=O)[nH]1</smiles><smiles></smiles>

Fig. (1). Molecular structures [7] of thymine (T), adenine (A), cytosine (C), guanine (G) and uracil (U). Bond length data [4-7] in Tables 1 and $\mathbf{2}$ correspond to distances between two adjacent (numbered) atoms.

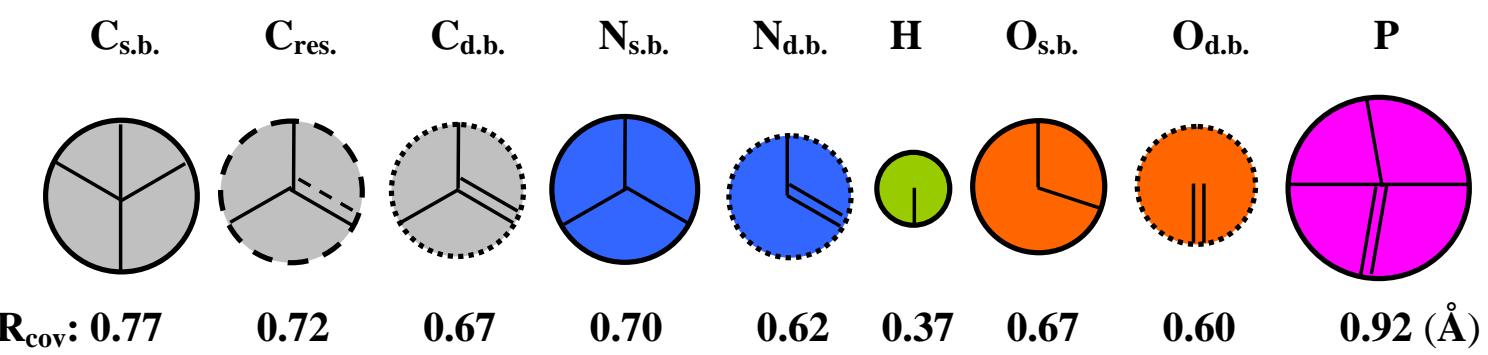

Fig. (2). The (nine) covalent radii $\mathrm{R}_{\mathrm{cov}}(+/-0.02 \AA)$ [4] of the five atoms constituting the molecular components of nucleic acids. C: gray, N: blue, $\mathrm{O}$ : orange, $\mathrm{H}$ : green and $\mathrm{P}$ : pink. Nature makes unique combinations of these five atoms (from mono to pentavalent) into the molecules of life (see Figs. (4-6)).

\section{BOND LENGTHS IN ALL THE ABOVE MOLECULES AS SUMS OF ATOMIC COVALENT RADII}

The above additivity of atomic radii was then tested for the distances between any two atoms in the skeletal structures of the molecular components of nucleic acids by comparing the data from [4-7] with the sums R(sum) of the appropriate atomic covalent radii in Fig. (2). The data for $A, G$, $\mathrm{T}$ and $\mathrm{C}$ are given in Table $\mathbf{1}$ and for $\mathrm{U}, \mathrm{Ri}, \mathrm{De}$ and $\mathrm{Ph}$ in Table 2. The average values of the bond lengths [4-7] for the above molecules (from Tables 1 and 2) and the corresponding $\mathrm{R}$ (sum) for the various bonds are tabulated in Table $\mathbf{3}$. On plotting these bond lengths $v s \mathrm{R}$ (sum) as shown in Fig. (3), and drawing a least square straight line through all the data points, it was found to have a slope of 1.01 and an intercept of -0.02 . On using this slope and intercept, the calculated bond lengths (given in the last column in Table 3 ) are found to be identical with R(sum) (col. 2, Table 3). This shows that the bond lengths in all the above molecules can be considered as the sums of the relevant atomic covalent radii.

Note also that since this graph includes the theoretical values of bond lengths from [6], the above result shows that the latter values are good representations of the radii sum, R(sum).

The above finding has thus enabled the conventional molecular structures (see Fig. (1)) to be resolved (for the first time) into the atomic structures based on the individual covalent radii of the constituent atoms. Fig. (4) shows the atomic structures of the bases: T (U), A, C and G and Fig. (5), those for phosphoric acid, deoxyribose (and ribose). For these molecules, while confirming Pauling's [4] two $\mathrm{CN}$ bonds of lengths 1.32 and $1.36(+/-0.02 \AA)$ and one $\mathrm{CC}$ bond length of $1.40 \AA$, it is found here that actually there are five $\mathrm{CN}$ bonds, three $\mathrm{CC}$ bonds, two $\mathrm{CO}$ bonds and two PO bonds (see Table 3).

The bond lengths of $\mathrm{C}, \mathrm{O}$ and $\mathrm{N}$ with $\mathrm{H}\left(\mathrm{R}_{\mathrm{cov}}=0.37 \AA\right.$, see Fig. (2)) are also given in Table 3. Note that whereas the increase in the $\mathrm{CH}$ bond length in going from aromatic to aliphatic $\mathrm{C}$ is attributed by Pauling [4] to the change in the radius of $\mathrm{H}$, here it is accounted for by those of $\mathrm{C}$.

Fig. (6) shows how a combination of all the molecules in Figs. $(\mathbf{4 , 5})$ fits into the known 34:20 $\AA$ dimension of the DNA nucleotides and a detailed description is given in the caption for this figure.

Support for the additivity of covalent atomic radii in bond lengths has been found also for the bonds in xanthine, caffeine and related compounds [3c].

Thus, it is concluded here that the skeletal bond lengths in the conventional molecular structures of the components of DNA and RNA are composed of the covalent radii (see Fig. (2)) of the two adjacent atoms constituting the bonds (see Figs. $(4,5))$.

\section{ACKNOWLEDGEMENTS}

The author is grateful to Prof. E. Palecek of the Institute of Biophysics for the moral support, and for the grants AVOZ50040507 of the Academy of Sciences of the Czech Republic and LC06035 of the Ministry of Education, Youth and Sports of the Czech Republic. 


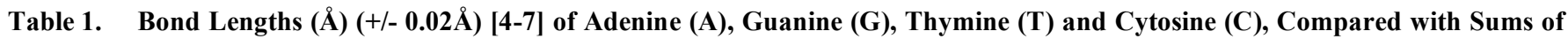
Atomic Covalent Radii, R(sum) (Upper Values, in Bold, See Also Table 3 and Fig. (4))

\begin{tabular}{|c|c|c|c|c|c|c|c|}
\hline Adenine & Bond Lengths & Guanine & Bond Lengths & Thymine & Bond Lengths & Cytosine & Bond Lengths \\
\hline N1-C2 & $1.34=0.62+0.72$ & N1-C2 & $1.37=0.67+0.70$ & N1-C2 & $1.37=0.67+0.70$ & $\mathrm{~N} 1-\mathrm{C} 2$ & $1.37=0.67+0.70$ \\
\hline$*$ & $1.34,1.34,1.32,1.34$ & & $1.32,1.37,1.34$ & & $1.38,1.38,1.36$ & & $1.41,1.40,1.36$ \\
\hline C2-N3 & $1.34=0.62+0.72$ & $\mathrm{C} 2-\mathrm{N} 3$ & $1.29=0.67+0.62$ & $\mathrm{C} 2-\mathrm{N} 3$ & $1.37=0.67+0.70$ & $\mathrm{C} 2-\mathrm{N} 3$ & $1.37=0.67+0.70$ \\
\hline$*$ & $1.34,1.33,1.32,1.34$ & & $1.36,1.32,1.32$ & & $1.38,1.37,1.36$ & & $1.37,1.35,1.34$ \\
\hline N3-C4 & $1.34=0.62+0.72$ & $\mathrm{~N} 3-\mathrm{C} 4$ & $1.29=0.67+0.62$ & $\mathrm{~N} 3-\mathrm{C} 4$ & $1.37=0.67+0.70$ & $\mathrm{~N} 3-\mathrm{C} 4$ & $1.29=0.67+0.62$ \\
\hline$*$ & $1.34,1.34,1.36,1.34$ & & $1.42,1.35,1.32$ & & $1.40,1.38,1.36$ & & $1.31,1.34,1.32$ \\
\hline $\mathrm{C} 4-\mathrm{C} 5$ & $1.39=0.67+0.72$ & $\mathrm{C} 4-\mathrm{C} 5$ & $1.39=0.67+0.72$ & $\mathrm{C} 4-\mathrm{C} 5$ & $1.39=0.67+0.72$ & $\mathrm{C} 4-\mathrm{C} 5$ & $1.39=0.67+0.72$ \\
\hline$*$ & $1.40,1.38,1.40,1.44$ & & $1.44,1.38,1.38$ & & $1.46,1.45,1.40$ & & $1.44,1.43,1.40$ \\
\hline C5-C6 & $1.39=0.67+0.72$ & C5-C6 & $1.39=0.67+0.72$ & C5-C6 & $1.39=0.67+0.72$ & C5-C6 & $1.39=0.67+0.72$ \\
\hline$*$ & $1.39,1.41,1.40,1.38$ & & $1.39,1.42,1.40$ & & $1.35,1.34,1.40$ & & $1.36,1.34,1.40$ \\
\hline C6-N1 & $1.34=0.62+0.72$ & C6-N1 & $1.37=0.67+0.70$ & C6-N1 & $1.37=0.67+0.70$ & C6-N1 & $1.37=0.67+0.70$ \\
\hline$*$ & $1.34,1.35,1.32,1.34$ & & $1.36,1.39,1.36$ & & $1.37,1.38,1.36$ & & $1.35,1.37,1.34$ \\
\hline C5-N7 & $1.29=0.67+0.62$ & C5-N7 & $1.34=0.62+0.72$ & $\mathrm{C} 2-\mathrm{O} 2$ & $1.27=0.67+0.60$ & $\mathrm{C} 2-\mathrm{O} 2$ & $1.27=0.67+0.60$ \\
\hline$*$ & $1.37,1.39,1.32,1.32$ & & $1.37,1.39,1.32$ & & $1.22,1.22,1.23$ & & $1.22,1.24,1.23$ \\
\hline N7-C8 & $1.29=0.67+0.62$ & $\mathrm{~N} 7-\mathrm{C} 8$ & $1.29=0.67+0.62$ & $\mathrm{C} 4-\mathrm{O} 4$ & $1.27=0.67+0.60$ & C4-N4 & $1.37=0.67+0.70$ \\
\hline$*$ & $1.32,1.31,1.32,1.28$ & & $1.32,1.31,1.32$ & & $1.22,1.23,1.23$ & & $1.36,1.34,1.35$ \\
\hline C8-N9 & $1.37=0.67+0.70$ & C8-N9 & $1.37=0.67+0.70$ & C5-M5 & $1.49=0.72+0.77$ & N1-C1' & $1.47=0.70+0.77$ \\
\hline$*$ & $1.37,1.37,1.33,1.37$ & & $1.37,1.37,1.32$ & & $1.49,1.50,1.53$ & & $-, 1.47,1.53$ \\
\hline N9-C4 & $1.42=0.70+0.72$ & N9-C4 & $1.37=0.67+0.70$ & $\mathrm{~N} 1-\mathrm{C} 1{ }^{\prime}$ & $1.47=0.70+0.77$ & & \\
\hline$*$ & $1.38,1.37,1.36,-$ & & $1.37,1.38,1.34$ & & $-, 1.47,1.53$ & & \\
\hline C6-N6 & $1.42=0.70+0.72$ & $\mathrm{C} 2-\mathrm{N} 2$ & $1.37=0.67+0.70$ & & & & \\
\hline$*$ & $-, 1.34,1.35,1.34$ & & $1.38,1.34,1.35$ & & & & \\
\hline N9-C1' & $1.47=0.70+0.77$ & C6-O6 & $1.27=0.67+0.60$ & & & & \\
\hline$*$ & $-, 1.46,1.53,-$ & & $1.22,1.24,1.23$ & & & & \\
\hline & & N9-C1' & $1.47=0.70+0.77$ & & & & \\
\hline$*$ & & & $-, 1.46,1.53$ & & & & \\
\hline *Values: & & *Values: & & *Values: & & *Values: & \\
\hline \multicolumn{2}{|c|}{ Refs. $6,7,4,5$} & \multicolumn{2}{|r|}{ Refs. $6,7,4$} & Refs. $6,7,4$ & & Refs. $6,7,4$ & \\
\hline
\end{tabular}

Table 2. Bond Lengths (+/- 0.02Å) [4-7] of Uracil (U), Ribose (Ri), Deoxyribose (De), Phosphoric Acid/Phosphate (Ph), Compared with Sums of Atomic Covalent Radii, R(sum) (Upper Values, in Bold, See Also Table 3 and Figs. $(4,5)$

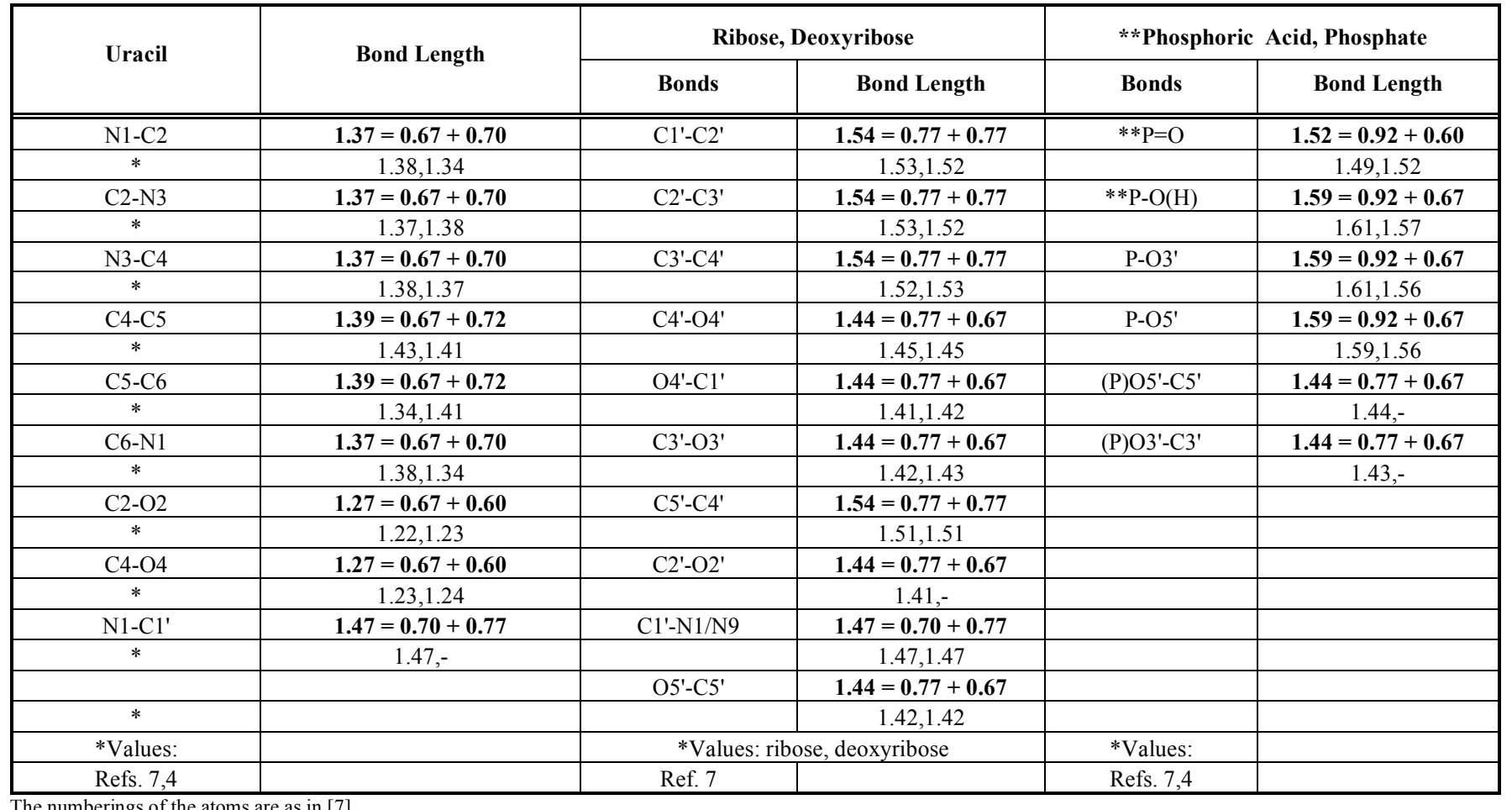


Table 3. Average Bond Lengths (+/- 0.03 Å) [4-7] Corresponding to Sums of Atomic Covalent Radii, R(sum)

\begin{tabular}{|c|c|c|c|c|c|c|c|c|c|}
\hline Bonds & R(sum) & $\mathbf{A}$ & $\mathbf{G}$ & $\mathbf{T}$ & $\mathbf{C}$ & $\mathbf{U}$ & Ri, De & $\mathbf{P h}$ & $\frac{\text { Calculated }}{\text { Bond Length }}$ \\
\hline$C_{\text {d.b }}-\mathbf{N}_{\text {d.b }}$ & 1.29 & 1.33 & 1.32 & & 1.32 & & & & 1.29 \\
\hline $\mathrm{C}_{\text {res }}-\mathbf{N}_{\text {d.b }}$ & 1.34 & 1.34 & 1.37 & & & & & & 1.34 \\
\hline$C_{d . b}-N_{s . b}$ & 1.37 & 1.36 & 1.36 & 1.37 & 1.36 & 1.37 & & & 1.37 \\
\hline$C_{s . b}-O_{s . b}$ & 1.44 & & & & & & 1.42 & 1.44 & 1.44 \\
\hline$C_{\text {s.b }}-N_{\text {s.b }}$ & 1.47 & 1.5 & 1.5 & 1.45 & 1.5 & 1.47 & 1.47 & & 1.47 \\
\hline $\mathrm{C}_{\text {s.b }}-\mathrm{C}_{\text {res }}$ & 1.49 & & & 1.48 & & & & & 1.49 \\
\hline $\mathbf{P}-\mathbf{O}_{\text {d.b }}$ & 1.52 & & & & & & & 1.51 & 1.52 \\
\hline$C_{\text {s.b }}-C_{\text {s.b }}$ & 1.54 & & & & & & 1.52 & & 1.54 \\
\hline $\mathbf{N}_{\text {s.b }}-\mathbf{H}$ & \multicolumn{2}{|l|}{$\mathbf{1 . 0 7}(\mathrm{A}, \mathrm{T}, \mathrm{U}, \mathrm{C}, \mathrm{G})$} & & & & & & & \\
\hline $\mathbf{O}_{\text {s.b }}-\mathbf{H}$ & \multicolumn{2}{|l|}{$1.04(\mathrm{Ri}, \mathrm{DE}, \mathrm{Ph})$} & & & & & & & \\
\hline
\end{tabular}

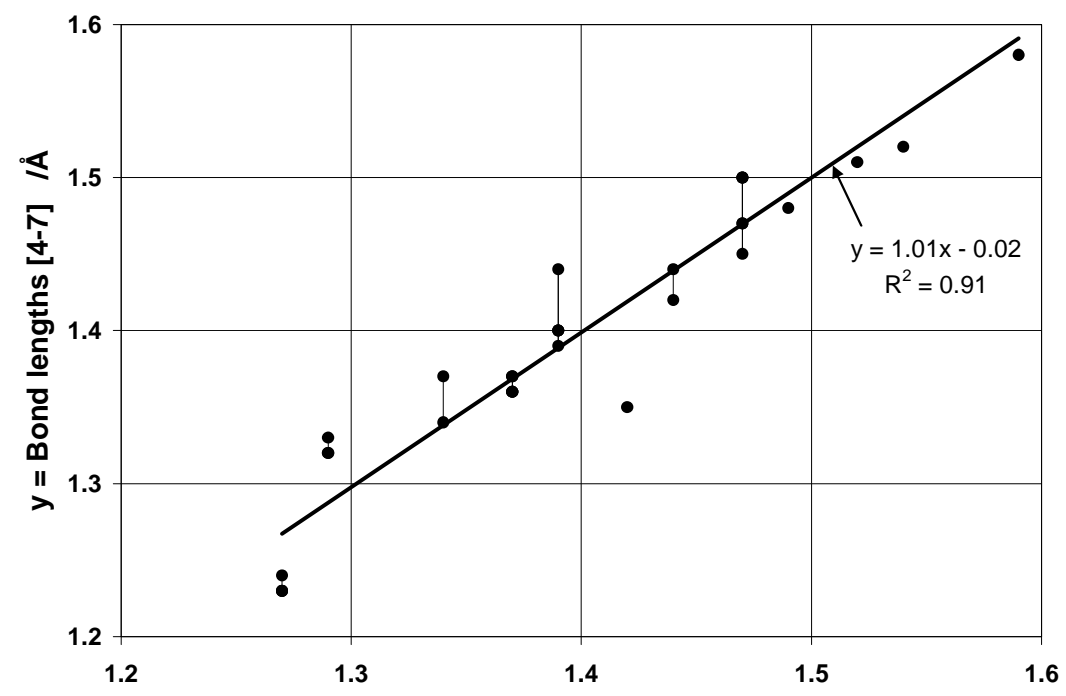

$\mathrm{x}=$ Sums of atomic radii, $\mathbf{R}($ sum) $/ \AA$

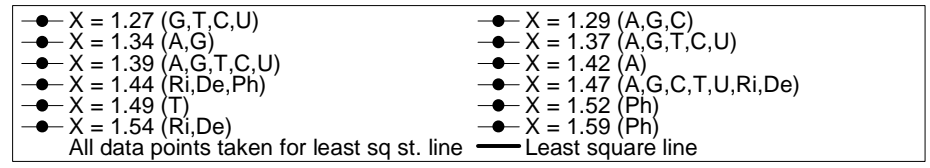

Fig. (3). Graph of the bond lengths from [4-7] vs the sums of atomic covalent radii, R(sum), data in Table 3. The unit slope and zero intercept of the least squares line show that the bond lengths are equal to R(sum). 
Thymine (T) [Uracil (U) has $\mathrm{H}$ at $\mathrm{C}(5)$ ]
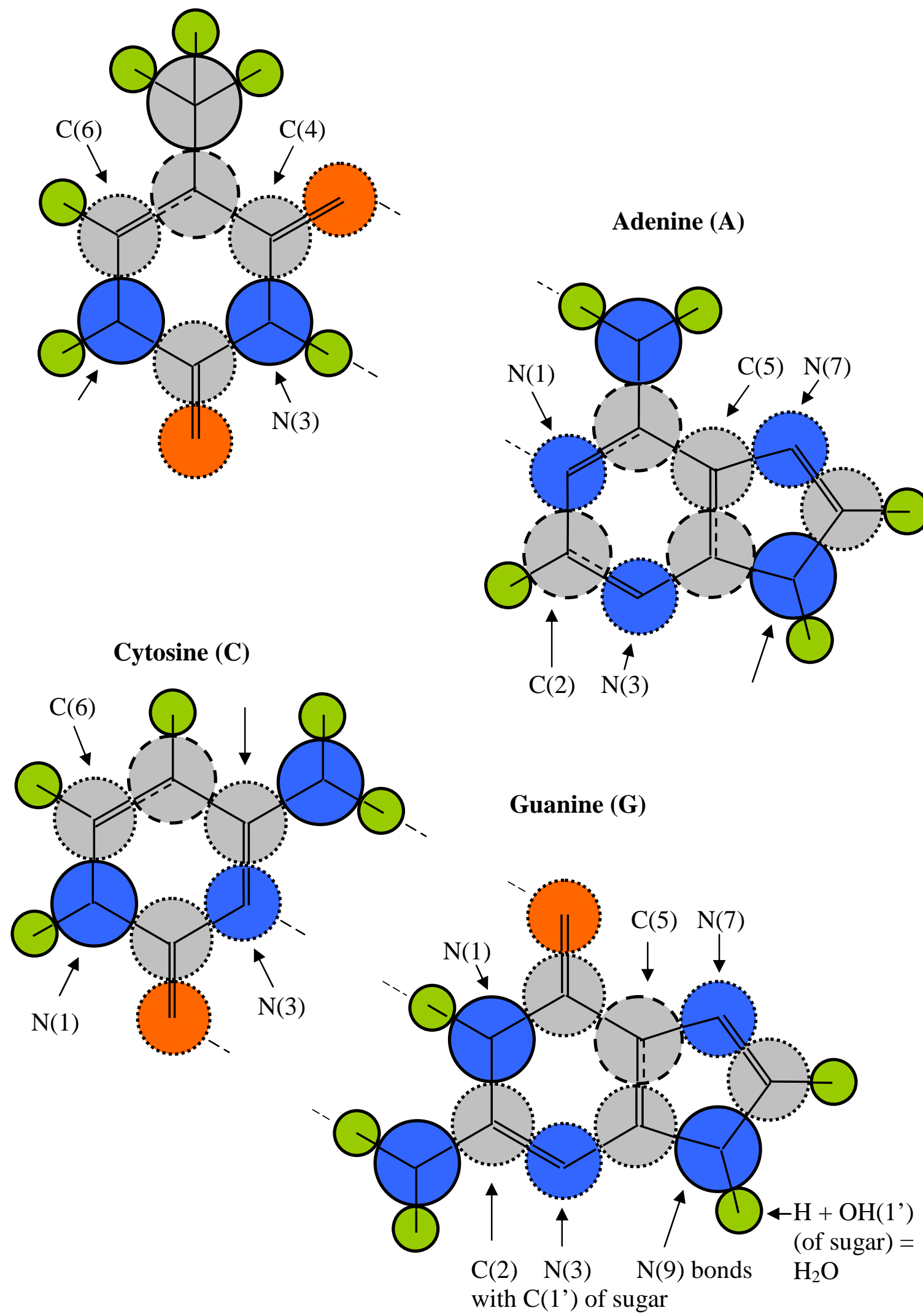

Fig. (4). Atomic structures of the purines: $A$ and $G$ and pyrimidines: $T$, (U) and C. All bond lengths are sums of the covalent radii of the adjacent atoms (see Table 3 ). Base pairing [1 a, b] occurs when hydrogen bonds connect $\mathrm{T}$ with $\mathrm{A}$, and $\mathrm{C}$ with $\mathrm{G}$ along the broken lines, with bond lengths as in [3b]. 


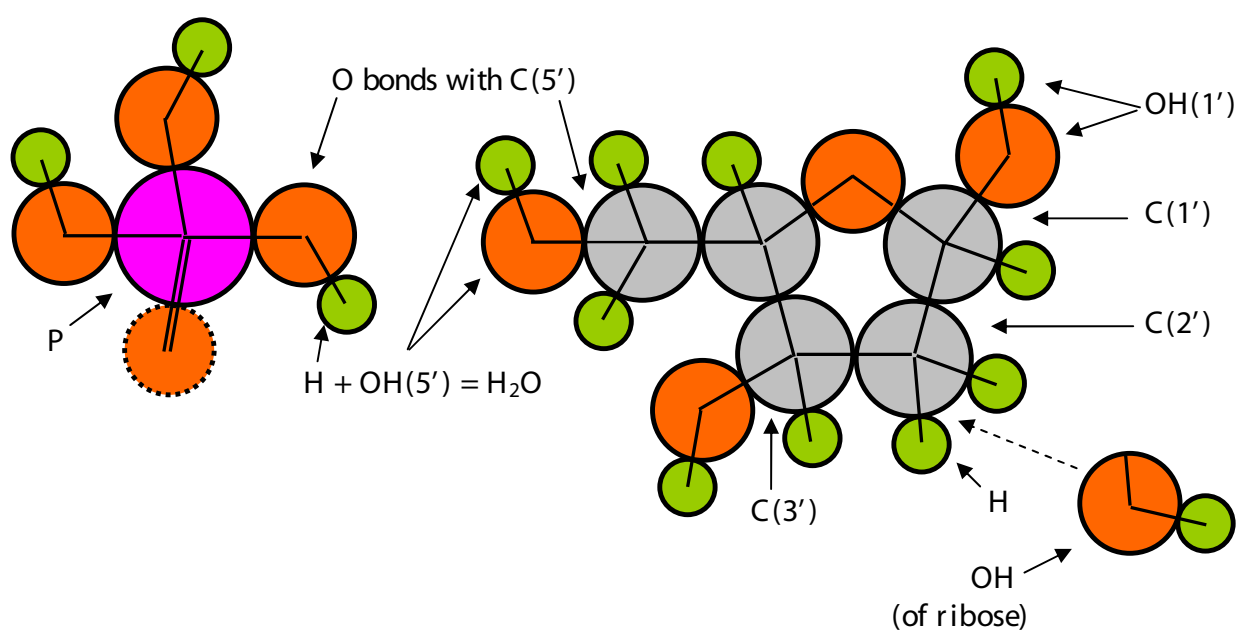

Fig. (5). Atomic structures of phosphoric acid and deoxyribose (and ribose). All bond lengths are sums of the covalent radii of the adjacent atoms (see Table 3). By the elimination of water molecules as shown, the bases combine with sugars and the latter with phosphoric acid to form nucleotides [4-7].

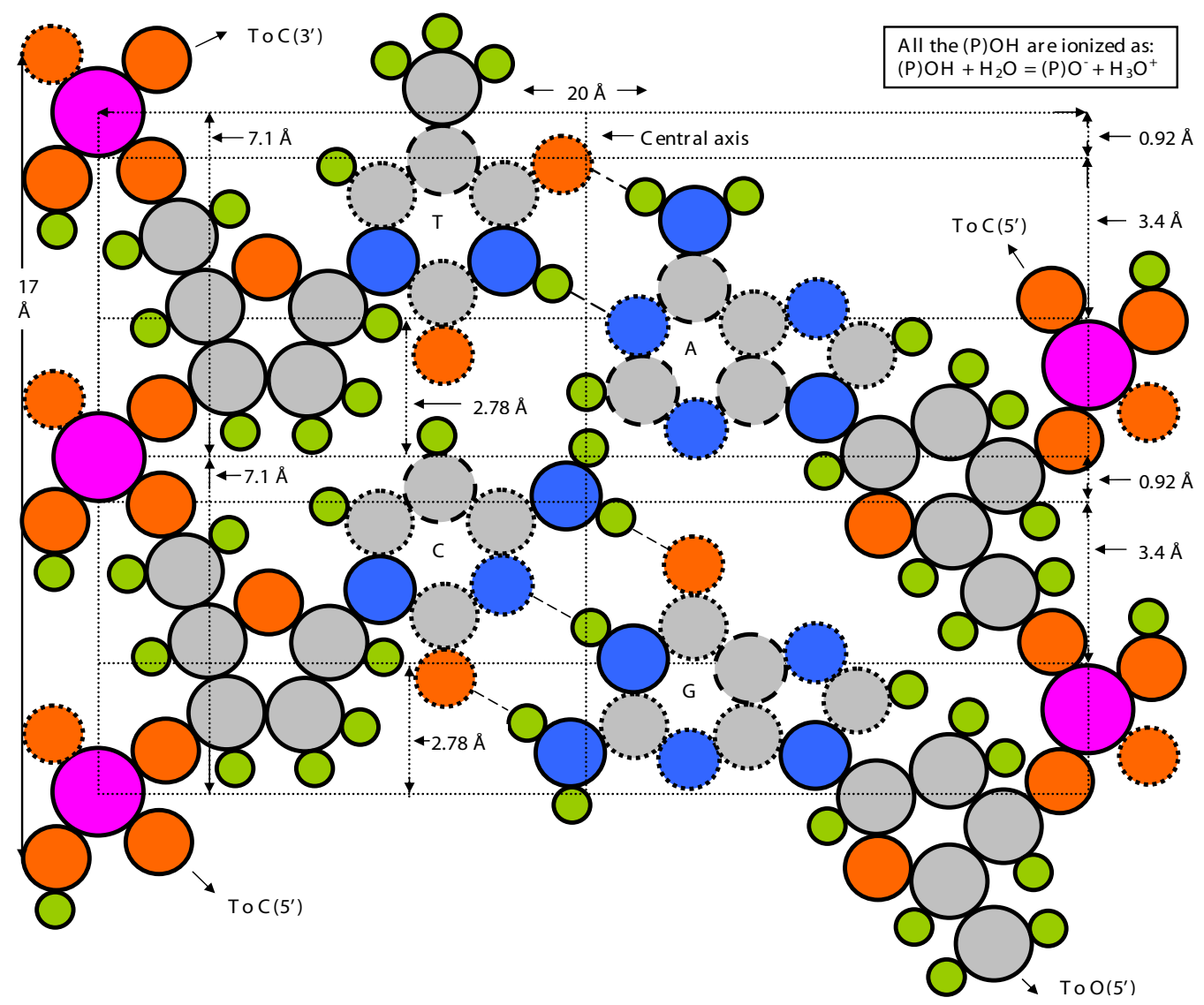

Fig. (6). Atomic structures of the DNA nucleotides with the Watson-Crick base pairs (T, A and C, G connected by H-bonds as shown) attached to deoxyribose and phosphate molecules. As per the known crystallographic distances [1c], the two $\mathrm{P}$ atoms (pink) attached via $\mathrm{O}\left(5^{\prime}\right.$ ) and $\mathrm{O}\left(3^{\prime}\right)$ (orange) to the sugar molecules are $7.1 \AA$ apart and the distance of each P atom from the central axis is $10 \AA$. The base pairs are perpendicular to the plane of the sugar-phosphate back bone and form the steps of the ladder of the double helix. The steps are $3.4 \AA$ apart [1c]. The distance of $17 \AA$ containing $5 \mathrm{P}$ atoms is half of the $34 \AA$ turn (with $10 \mathrm{P}$ atoms) of the helix and the distance $2.78 \AA$ can be considered as the thickness of the steps. (For the colors, radii and bonds of the atoms see Figs. $(\mathbf{2}, \mathbf{4 , 5})$ ).

Note: The above Figure holds also for RNA, but with Uracil (U) and Ribose in place of Thymine (T) and deoxyribose respectively. 


\section{REFERENCES}

[1] Watson JD, Crick FHC. a) A structure for deoxyribonucleic acid. Nature 1953; 171: 737-738 and b) Genetic implications of the structure of deoxyribonucleic acid. Nature 1953; 171: 964-967, c) Franklin RE, Gosling RG. Molecular configuration in sodium thymonucleate. Nature 1953; 171: 740-741; see for full papers: http://www.nature.com/nature/dna50/archive.html

[2] Heyrovska R. a) The Golden ratio, ionic and atomic radii and bond lengths. Mol. Phys. 2005; 103: 877-882 and b) The Golden ratio, atomic, ionic and molecular capacities and bonding distances in metallic hydrides. International Joint meeting of ECS, USA and Japanese, Korean and Australian Societies, Honolulu, Hawaii, 2004; Extd. Abs. C2-0551. http://www.electrochem.org/d1/ma/206/ pdfs/0551.pdf.

[3] Heyrovska R. a) Dependences of ion-water distances on covalent radii, ionic radii in water and distances of oxygen and hydrogen of water from ion/water boundaries. Chem Phys Lett 2006; 429: 600$605, b)$ Dependence of the length of the hydrogen bond on the covalent and cationic radii of hydrogen, and additivity of bonding distances. Chem Phys Lett 2006; 432: 348-351. c) Heyrovska R, Narayan S. $10^{\text {th }}$ Eurasia conference on chemical sciences, Manila, Philippines, 7-11 January 2008, Book of Abstracts, p. 330.

[4] a) Pauling L. The Nature of the Chemical Bond. Cornell Univ. Press, Ithaca, New York, 1960; b) http://wps.prenhall.com/wps/ media/objects/3311/3390919/blb0702.html

[5] Lehninger AL. Biochemistry. Worth Publishers, Inc., New York, 1975.

[6] Preuss M, Schmidt G, Seino K, et al. Ground- and excited-state properties of DNA base molecules from plane-wave calculations using ultrasoft psuedopotentials. J Comput Chem 2004; 25: 112122.

[7] Nucleic acid database. http://ndbserver.rutgers.edu. 